\section{PTU-59 A QUALITY IMPROVEMENT PROJECT (QIP) TO REDUCE INAPPROPRIATE BIOPSIES AT ENDOSCOPY}

'Dinali Jayawardena*, ${ }^{2}$ loannis Koumoutsos, ${ }^{1}$ Ben Warner. 'Darent Valley Hospital, UK; ${ }^{2}$ Southend University Hospital, UK

\subsection{6/gutjnl-2021-BSG.132}

Introduction Histopathology costs represent a significant cost for endoscopy departments. Biopsies are useful when they can influence the management of our patients. Our aim was to demonstrate that application of a biopsy protocol could reduce costs and inappropriate use of resources.

Methods Analysis of 486 OGDs and 412 Colonoscopies was performed between April and May 2019. A biopsy protocol was produced using national guidelines (see Table 1). An assessment was made of the appropriateness of biopsy in each case. A price list of sample pots was obtained, and estimated costs were calculated to deduce the potential cost savings if guidelines had been adhered to over this 2 -month period.

Results Of the 486 OGDs performed, biopsies were taken in 185 cases, of which 131 were appropriate (71\%). An estimated $£ 1816$ may have been saved from reducing inappropriate biopsies. Of the 412 Colonoscopies performed, biopsies were taken in 266 cases, of which 190 were appropriate (71\%). An estimated $£ 2555$ may have been saved from reducing inappropriate biopsies over this 2 -month period.

\begin{tabular}{l} 
Abstract PTU-89 Table 1 Sample of biopsy protocol for \\
Oesophagus and Colon. Guidance was also created for Gastric/ \\
Duodenal biopsies \\
\hline OESOPHAGUS \\
\hline YES
\end{tabular}

Conclusions Encouraging adherence to biopsy protocols reduces the number of inappropriate biopsies, pathology workload and costs. A learning event was held to disseminate the protocol and the results of this QIP to our endoscopists.

\section{PTU-60 THE PREVALENCE OF SERRATED POLYPS IN A MULTI- ETHNIC SOCIETY IN THE UNITED KINGDOM}

Mohamed G Eisa*, Roshini Kurian, Peter Wurm, Richard Robinson. University Hospitals of Leicester, Leicester, UK

\subsection{6/gutjnl-2021-BSG.133}

Introduction Leicestershire is the 16th most populous county in the United Kingdom. The estimated population is around $706,155^{1}$. It has a multi-ethnic diverse group where non-white comprises $15 \%$ of its population ${ }^{1}$. The majority of the nonwhite is South Asian.

Aim We aim to assess the prevalence of serrated polyps, defined as hyperplastic polyp (HP), sessile serrated lesion (SSL), traditional serrated adenoma (TSA) or mixed polyp ${ }^{2}$, in this multi-ethnic group in patients attending for Bowel Cancer Screening (BCS) colonoscopy.

Methods We carried out a retrospective observational study of all serrated lesions (SLs) over a two-year period between 1st May 2018 to 30th April 2020.

Patients' demographic, polyp morphology \& site were obtained from our Bowel Cancer Screening Programme (BCSP) database.

The self-reported ethnic origin of all individuals was recorded.

Non-serrated lesions (i.e tubular adenoma, tubulovillous adenoma and villous adenoma) were excluded from the study.

For statistical analysis we used Pearson Chi-square, independent sample T-test and one-way analysis of variance were used as appropriate. $\mathrm{P}$ value of 0.05 was considered statistically significant.

All analysis was conducted using SPSS version 26.

Results Over a 2 year-period, 2264 screening/surveillance colonoscopies were carried out, of which 493 patients had one or more SLs.

The mean age was 67.6 years, and $66.5 \%$ of patients were male.

The white Caucasian comprised $87.2 \% \quad(n=430)$ of the study population while the Asian group was $12.8 \%(n=63)$.

The frequency of polyps as per morphology was as following: hyperplastic $82.4 \%(\mathrm{n}=406)$, sessile serrated lesion $10.5 \%$ $(\mathrm{n}=52)$, traditional serrated lesion $1.2 \% \quad(\mathrm{n}=6)$ and mixed type $5.9 \%(n=29)$.

In the white group: $80.2 \%(n=345)$ of polyps were hyperplastic, $11.7 \%(n=50)$ were SSL, 1.4\% $(n=6)$ was TSA and $6.7 \%(n=29)$ were mixed type.

In the Asian group: 96.8\% $(n=61)$ of polyps were hyperplastic, $3.2 \%(n=2)$ were SSL and none was TSA or mixed type.

The mean number of SLs per individual was significantly higher in white Caucasian than in Asians, 1.53 (SD 1.10) vs. 1.23 (SD 0.49), $(\mathrm{p}=0.001)$.

Between the two groups, the prevalence of SSL and the mixed type were higher in the white group $(p=0.001$ and $\mathrm{p}=0.003$, respectively).

On the one-way analysis, the mixed type was more prevalent amongst the different types of SLs $(p=0.001)$.

The site of SLs in the colorectum, in order from highest to lowest, was as following: Sigmoid 53.6\% $(\mathrm{n}=266)$, Rectum 25\% ( $\mathrm{n}=124)$, Ascending 23.5\% ( $\mathrm{n}=117)$, Transverse 20.9\% 